\section{The trochanteric fat pad}

P. Panettiere, ${ }^{1}$ D. Accorsi, ${ }^{1}$ L. Marchetti, ${ }^{1}$ A.M. Minicozzi, ${ }^{2}$ G. Orsini, ${ }^{3}$ P. Bernardi, ${ }^{4}$ D. Benati, ${ }^{4}$ G. Conti, ${ }^{4}$ A. Sbarbati ${ }^{4}$

'Dipartimento di Scienze Chirurgiche Specialistiche ed Anestesiologiche, University of Bologna;

2First Division of General Surgery,

University of Verona;

${ }^{3}$ Department of Clinical Sciences and

Stomatology, Polytechnic University

of Marche, Ancona;

${ }^{4}$ Human Anatomy and Histology Section, University of Verona; Italy

\section{Abstract}

Technological developments based on the use of autologous white adipose tissue (WAT) attracted attention to minor fat depots as possible sources of adipose tissue. In plastic surgery, the trochanteric fatty pad is one of the most used WAT depots for its location and organoleptic characteristics that make it particularly suitable for reconstructive procedures. Despite its wide use in clinic, the structure of this depot has never been studied in detail and it is not known if structural differences exist among trochanteric fat and other subcutaneous WAT depots. The present study was performed on trochanteric fat pad with the aim to clarify the morphology of its adipocytes, stroma and microcirculation, with particular reference to the stem niches. Histological and ultrastructural studies showed that the main peculiar feature of the trochanteric fat concerns its stromal component, which appears less dense than in the other subcutaneous WATs studied. The intraparenchymal collagen stroma is poor and the extracellular compartment shows large spaces, filled with electron-light material, in which isolated collagen bundles are present. The adipocytes are wrapped in weak and easily detachable collagen baskets. These connective sheaths are very thin compared to the sheaths in other subcutaneous WAT depots. The capillaries are covered by large, long and thin elements surrounded by an external lamina; these perivascular cells are poor in organelles and mainly contain poly-ribosomes. In conclusion, when compared to other WAT deposits, the trochanteric fatty pad shows structural peculiarities in its stroma and microcirculation suggesting a high regenerative potential. Resistance, dissociability, microvascular weft and high regenerative potential make the trochanteric fatty pad a privileged source for harvesting in autologous WAT-based regenerative procedures.

\section{Introduction}

In regenerative medicine, the methods based on autologous subcutaneous white adipose tissue (WAT) transfer are widely used. ${ }^{1-4}$ In regenerative surgical procedures using autologous WAT, the choice of the donor site is generally based on empirical considerations, ${ }^{5}$ due to the absence of specific data about the different sites, obtained with modern methods of WAT analysis. ${ }^{6-9}$ Data concerning the choice of the donor site should derive from the follow up of clinical trials, but knowledge of the different types of WATs could be relevant in the setting up of a harvesting protocol for the correction of the different pathologies. ${ }^{10}$ At the present, data about each single fat depot are scarce. ${ }^{11-15}$ In addition, the knowledge about adipose stem niches, which play an important role in the healing processes based on autologous WAT, ${ }^{16}$ is particularly defective. There are no specific studies on the microvascular compartments containing the niches in the fat depots that are common WAT donor sites (e.g. the trochanteric fatty pad or other WAT depots in the limbs). It is generally believed that there are no significant morphologic differences between these depots and the abdominal subcutaneous WAT, even if no comparative study specifically aimed at verifying such features has ever been performed. As a matter of fact, while the abdominal WAT represents essentially a site of triglycerides storing, the limbs depots also play mechanical functions as they are often associated to articular interlines or sites of particular mechanic stress. Possible morphologic differences in the adipocyte, microvascular or stem compartment could be related to different functional roles and biochemical differences among the fat depots. The stem cell compartment could be especially sensitive to the cellular turnover problems aroused by stress elements on the delicate adipose cells.

Among the depots of WAT located in the limbs, the trochanteric fatty pad is of great interest for several reasons. ${ }^{17}$ First of all, it plays such a high aesthetic role, especially in female individuals, to often require plastic surgery; in addition, it is one of the privileged sources of autologous WAT and can be considered a common donor site. ${ }^{18}$ This depot can be considered as a true organ with clear anatomic delimitations and a vascular peduncle. This depot has a mechanic function linked to hip motility and the trochanteric shear stress exclude its role as a mere metabolic storing site. Despite such reasons for interest, the studies about the trochanteric depots are very limited, and structural and ultrastructural data are totally absent. These data could be important also to explain the general biology of WAT,
Correspondence: Prof. Andrea Sbarbati, Anatomy and Histology Section, Medical Faculty, University of Verona, strada Le Grazie 8, 37134 Verona, Italy.

Tel. +39.045 .8027155 ; Fax: +39.045 .8027163$

E-mail: andrea.sbarbati@univr.it

Key words: fat transplantation, liposuction, adipose stem cells, adipocyte.

Received for publication: 10 January 2011 Accepted for publication: 1 April 2011

This work is licensed under a Creative Commons Attribution NonCommercial 3.0 License (CC BYNC 3.0).

\section{Copyright P. Panettiere et al., 2011}

Licensee PAGEPress, Italy

European Journal of Histochemistry 2011; 55:e16 doi:10.4081/ejh.2011.e16

which recently revealed unexpected complexity ${ }^{19-21}$ and plasticity, ${ }^{22,23}$ which must be taken into account in the perspective of possible complications. ${ }^{24}$ In particular, it would be interesting to elucidate why such depots do exhibit so profitable properties in adipose tissue transplantation. The present study was therefore performed on the trochanteric depots using light and electron microscopy in order to describe the morphology of the adipocytes and microcirculation, with particular reference to the stem niches.

\section{Materials and Methods}

Fat was harvested, after informed consent, from twelve female patients ranging in age from 45 to 60 years (average age: 52.7 years), who underwent fat harvesting from September to March 2010 for autologous fat implant. A mixture of $0.5 \%$ xylocaine + epinephrine $1: 200,000$ in saline solution was injected before suction. Fat harvesting was performed by a $3 \mathrm{~mm}$ cannula (1-hole, bullet tip) connected to a 10 cc syringe for vacuum; fat was then gently washed in saline and decanted. Centrifugation was not performed. In six patients, harvesting was performed from the trochanteric fatty pad. In six patients, control specimens were sampled from the flank area. As controls, we used WAT removed from other locations (abdomen $n=26$, flank $n=9$, thorax $\mathrm{n}=6$, limbs $\mathrm{n}=7$ ). For transmission electron microscopy (TEM), parts of the specimens were immediately fixed in $2.5 \%$ glutaraldehyde in Sorensen buffer pH 7.4 for $2 \mathrm{~h}$, postfixed in $1 \%$ osmium tetroxide in Sorensen buffer for 1 $\mathrm{h}$, dehydrated in graded acetones (60-100\%), embedded in Epon-Araldite and cut with an Ultracut E Ultramicrotome (Reichert, Wien, 
Austria). Ultrathin sections were stained with lead citrate and observed in an electron microscope Morgagni 268D (FEI-Philips, Eindhoven, The Netherlands). The semithin sections were stained with Toluidine blue. For scanning electron microscopy (SEM), the specimens were fixed in $2.5 \%$ glutaraldehyde in Sorensen buffer for $2 \mathrm{~h}$, postfixed in 1\% osmium tetroxide in Sorensen buffer for $1 \mathrm{~h}$, and dehydrated in graded acetone (Fluka); the specimens were then treated by a critical point dryer (CPD 030; Balzers Union Ltd., Balzers, Liechtenstein) and coated by gold, mounted on stubs and observed in an XL 30 ESEM (FEI-Philips). Data about adipocyte size were obtained during SEM examination using Scandium Sem Imaging Platform (Olympus).

\section{Results}

After removal, the pad is characterized by a high degree of fluidity and subsequent histological and ultrastructural evaluations were aimed at clarifying the tissutal basis of this organoleptic feature that makes this WAT deposit a preferred source for operation of adipose-based connective restructuring. Light and electron microscopy examinations showed that the trochanteric pad is composed of a peculiarly organized WAT. According to Sbarbati et al., ${ }^{15}$ it can be classified as a variety of structural WAT (sWAT), which is abundant in the limbs, thorax and flanks. As in the other types of sWAT, in the trochanteric pad as well an adipocyte compartment and a stromal compartment can be recognized (Figures 1,2,3 and 4). The adipocyte compartment mainly consists of mature adipose cells, whose mean diameter is $86.91 \pm 15.85$; these cells are slightly smaller than the adipocytes found in the large metabolic depots of the abdominal region $(97.69 \pm 15.61)$, although the difference found in the present study was not statistically significant. The morphology of the mature adipose cells of the trochanteric pad is similar as in the other sWAT depots (Figure 1, A-C): adipose cells show a thin rim of cytoplasm, in which micro-pinocytotic vesicles are the most common structures (Figure 1D).

The main characteristic of the trochanteric fat is surely represented by its stromal component (Figure 2). At light microscopy (Figure 1A), the stroma appears to be less dense than in the other sWATs located in the limbs or in the thorax; in the perivascular spaces among the adipocytes, the cellular elements are scarce (Figure 1B) and generally have a high nucleus-to-cytoplasm ratio and a cytoplasm poor in organelles. The intra-parenchymal collagen stroma (Figure $2 \mathrm{~A}, \mathrm{~B}$ ) is poor, and the extracellular compartment shows rather large spaces filled with light material, in which only collagen bundles and isolated cells are present. At TEM, the intercellular spaces appear to be filled with material characterized by very low
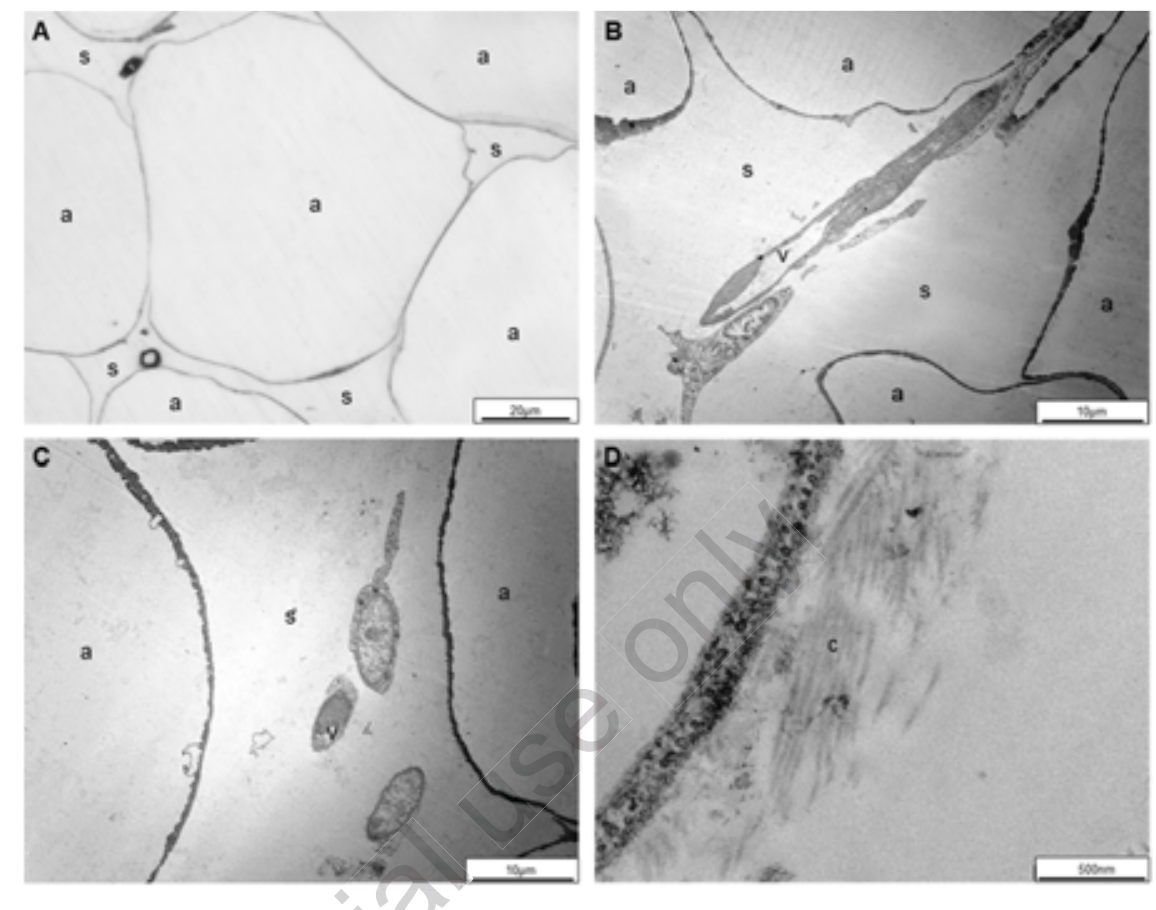

Figure 1. Human trochanteric white adipose tissue. (A) Light microscopy. (B-D) Transmission electron microscopy. The mesenchymal-like stroma is visible. Note the scarcity of collagen fibres and the presence of stromal elements. In panel D, isolated bundles of collagen fibers are present around an adipocyte. a, adipocyte; $v$, blood vessel; $s$, stromal connective; $c$, collagen fibers.
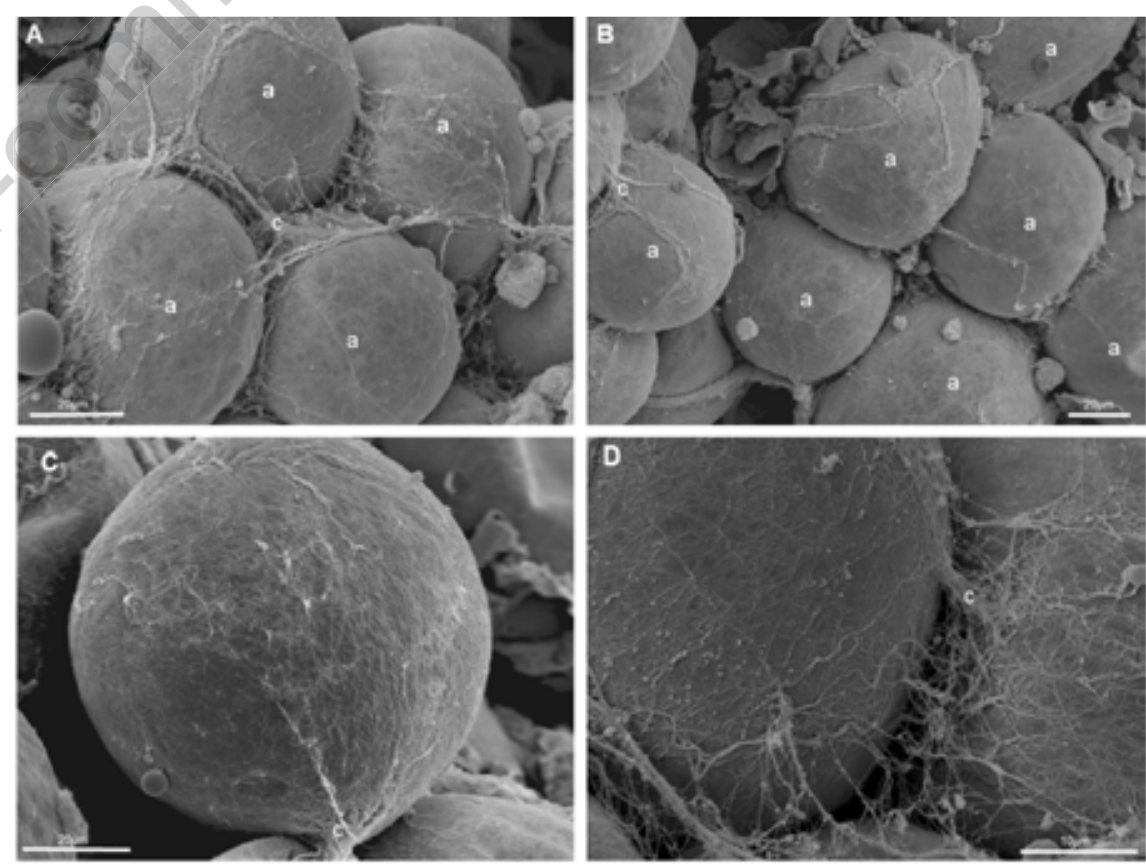

Figure 2. Human trochanteric white adipose tissue. Scanning electron microscopy. (A,B) at low magnification, collagen bundles in the stroma are visible; $(C)$ at medium enlargement, the basket around each adipocyte is visible; (D) at high magnification, a network of collagen fibers among the adipocytes is visible. a, adipocyte; c, collagen fibers. 
in which the adipocytes are embedded (Figure 2D).

The collagen bundles do not appears to be in particular relation with the blood capillaries; instead, they are often strictly associated to the adipocytes which are wrapped by a structure that has never been previously described and therefore deserves an accurate description. The mature adipocytes are covered by connective sheaths composed by a network of collagen fibers (Figures $2 \mathrm{C}$ and 3 ). In general, these connective sheaths are extremely thin when compared to the sheaths present in other subcutaneous adipose tissues. In fact, highresolution SEM images (Figure $3 \mathrm{C}, \mathrm{D}$ ) reveal that this structure, which could be called natural basket of the adipocytes, is composed by a very loose network of isolated collagen fibers in contact with the plasmalemma, as it can be easily observed at both TEM and SEM examinations. Moreover, the collagen basket that wraps these cells appears relatively easy to detach allowing the visualization of a smooth plasmalemma (Figure 3B). At TEM, the microvascular bed can also be studied (Figure 4). Capillary vessels are frequent and often show large lumina; they are surrounded by peculiar perivascular elements when compared to those in other subcutaneous adipose tissues. These cells are large, long and thin, and are surrounded by an external lamina; they often contact the vascular wall, but sometimes this connection is not visible; their cytoplasm contains few organelles among which polyribosomes are the most frequent. Moderate amounts of glycogen particles are visible in some cells. Thin elements with the morphologic features of pericytes were also observed surrounding the capillaries.

Control specimens, removed from deposits of WAT located in different regions (i.e. abdomen, flanks, thorax and limbs), revealed light and electron microscopic features according to those reported in the literature (Figure 5). When compared to the trochanteric pad, these adipose tissues are characterized by differences in particular in the stromal compartment. Briefly, the collagenic stroma and the natural basket of the adipocytes appear regularly thicker than those visible in the trochanteric pad. On the contrary, the walls of the microvascular elements are generally thinner and the capillary walls are generally made of endothelial cells only.

\section{Discussion}

To date, despite the well-known usefulness of the trochanteric fat pad in plastic surgery reconstructions, histological and ultrastructural studies about this depot are not available. In particular, it recently emerged the use of this tissue in regenerative procedures based on the Coleman method or its variations. ${ }^{1,25}$ In this case too, despite some anecdotic reports, to our knowledge no morphologic study has ever been performed before on the isolated or
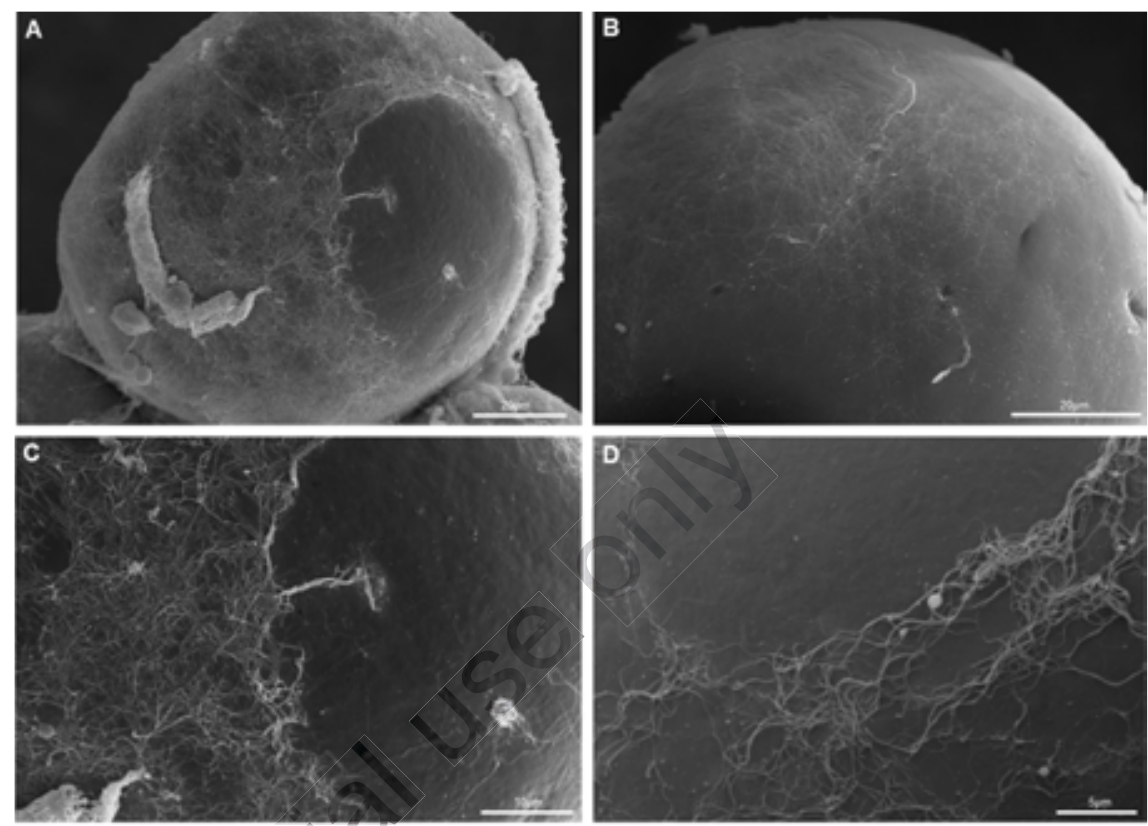

Figure 3. Human trochanteric white adipose tissue. Scanning electron microscopy. (A) an adipocyte surrounded by the periadipocytic basket is visible. The collagen fibers form an incomplete basket around the adipocytes; (B) the panel shows an adipocyte in which the periadipocytic basket has been removed. The plasma membrane appears to be regularly smooth; (C, D) high magnification of the periadipocytic basket. In these elements, the morphology of the basket, which is composed of a loose network of isolated collagen fibers is visible.
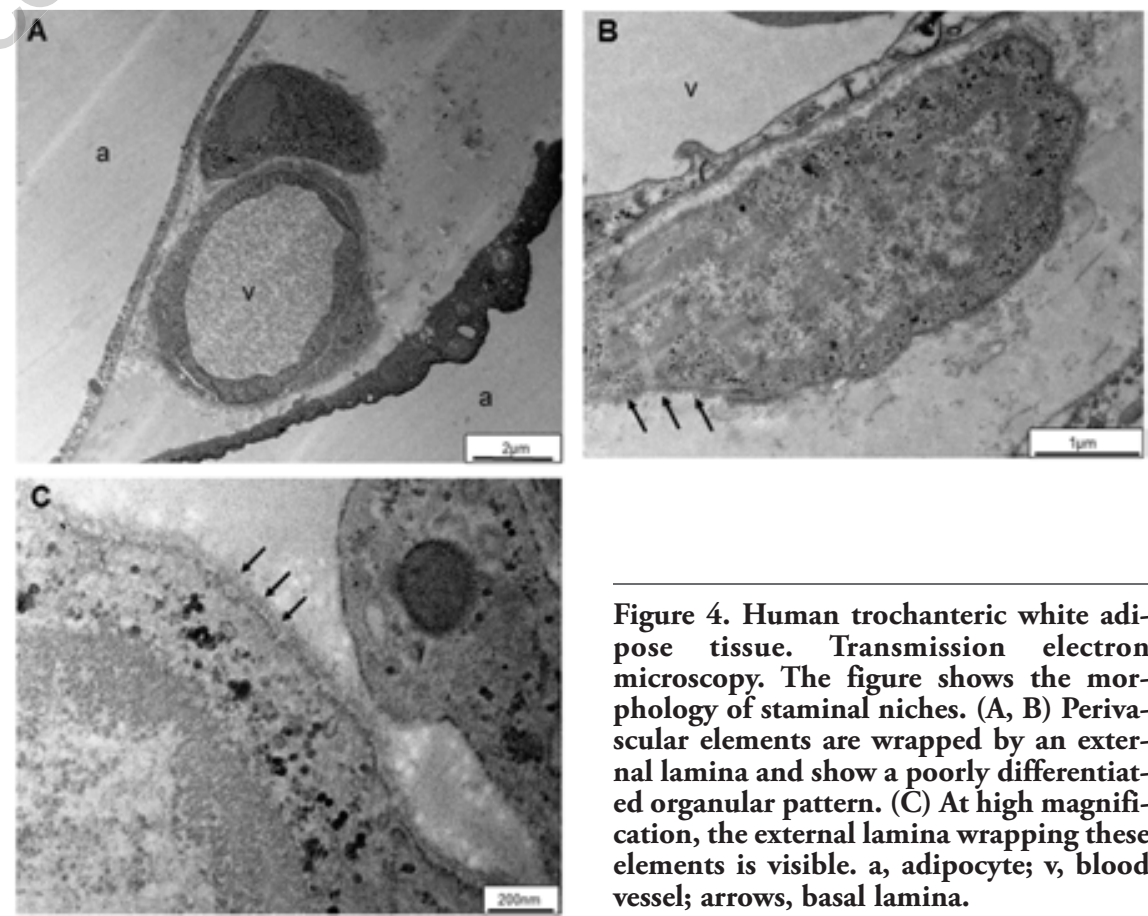

Figure 4. Human trochanteric white adipose tissue. Transmission electron microscopy. The figure shows the morphology of staminal niches. (A, B) Perivascular elements are wrapped by an external lamina and show a poorly differentiated organular pattern. (C) At high magnification, the external lamina wrapping these elements is visible. a, adipocyte; $v$, blood vessel; arrows, basal lamina. 
al studies allowed us to ascertain some interesting differences between this tissue and the subcutaneous WAT harvested from other sites and in particular from the abdominal area. In general, the trochanteric fat showed similarities with structural WATs, such as those located in other sites of the lower limb (e.g. the tight or the inner knee), while major differences were found with respect to the metabolic WAT found in the abdominal region.15 However, also when compared with the other structural WAT deposits, the trochanteric WAT displays some structural and ultrastructural characteristics that must be remarked.

In the trochanteric pad, the small size of the adipocytes and the morphology of the collagenic basket surrounding these cells could explain its vitality in the course of surgical isolation and transplant; moreover, adipocytes from the trochanteric WAT adhere loosely each other due to the thin network of the collagen in the stroma, and this explains why these cells may be easily dissociated.

Microcirculation is undoubtedly the most peculiar component of the trochanteric WAT. The capillaries are often covered by pericytic elements, often rich in cytoplasm, that overlap the endothelium: these duplications of the capillary basal membrane are often visible and suggest a high traffic through these elements toward the stroma. The microcirculation features suggest a structural sturdiness and a noteworthy regenerative potential linked to the richness in stem niches.

All these aspects (resistance, dissociability, sturdiness of the microvascular weft and high regenerative potential) suggest that trochanteric WAT might be a good harvesting source for autologous adipose tissue based procedures. Because of these specific feature of its microcirculation, it seems possible that the potential of differentiation of adiposederived stem cells isolated from the adipose tissue of the trochanteric fat pad might be particularly suited for regenerative procedures based on the use of autologous fat.

In conclusion, the main evidence emerging from the present work, i.e. the structural peculiarity of the trochanteric pad as compared to other WAT deposits and in particular to the abdominal fat, calls into question a concept of medicine that seemed to be untouchable: the structural identity of WAT. The idea that only one variant of subcutaneous WAT exists seems not to withstand when rigorous studies with adequate methodologies are performed. In surgical procedures, despite the fact that the two tissues are widely considered equivalent, the differences between abdominal and trochanteric WAT should not be underestimated. The data emerging from the present study should therefore be considered when approaching reconstructive procedures or
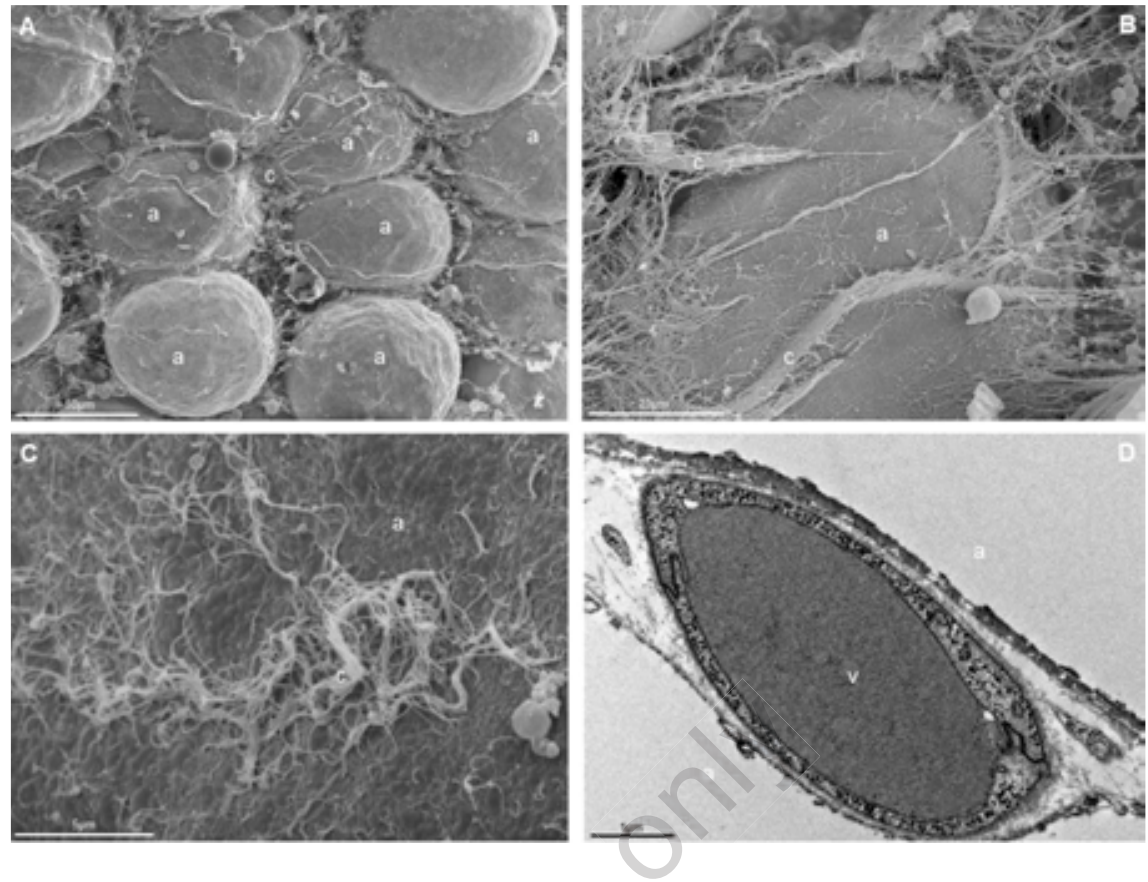

Figure 5. Human subcutaneous structural white adipose tissues. A-C, Scanning electron microscopy; the robust network of collagen fibers is visible at different magnifications; $D$, transmission electron microscopy; the thin wall of a capillary vessel is visible. a, adipocyte; v, blood vessel; c, collagen fibers.

transplants based on autologous adipose tissue. In this case, the material used is not only a common filler, but it is a living tissue employed to perform a true regeneration procedure, ${ }^{1,26}$ whose final result could depend on which tissue source had been selected. Therefore the harvesting site should not be chosen randomly, but based on evidence.

The present research suggests that follow up studies are required to assess possible differences between surgical procedures performed using WAT removed from different donor sites. In such a context, the knowledge of the differences among adipose tissues surely represents an important step toward the optimization of reconstructive procedures.

\section{References}

1. Coleman SR. Structural fat grafting: more than a permanent filler. Plast Reconstr Surg 2006;118:108S-120S.

2. Panettiere P, Marchetti L, Accorsi D, Del Gaudio GA. Aesthetic breast reconstruction. Aesthetic Plast Surg 2002;26:429-35.

3. Panettiere P, Marchetti L, Accorsi D. The serial free fat transfer in irradiated prosthetic breast reconstructions. Aesthetic Plast Surg 2009;33:695-700.

4. Rigotti G, Marchi A, Galiè M, Baroni G, Benati D, Krampera M, et al. Clinical treat- ment of radiotherapy tissue damage by lipoaspirate transplant: a healing process mediated by adipose-derived adult stem cells. Plast Reconstr Surg 2007;119:140922.

5. Pu LL. Discussion: Sources of processed lipoaspirate cells: influence of donor site on cell concentration. Plast Reconstr Surg 2008;122:619-20.

6. Lunati E, Marzola P, Nicolato E, Fedrigo M, Villa M, Sbarbati A. In vivo quantitative lipidic map of brown adipose tissue by chemical shift imaging at 4.7 Tesla. J Lipid Res 1999;40:1395-400.

7. Lunati E, Farace P, Nicolato E, Righetti C, Marzola P, Sbarbati A, et al. Polyunsaturated fatty acids mapping by (1)H MRchemical shift imaging. Magn Reson Med 2001;46:879-83.

8. Lunati E, Marzola P, Nicolato E, Sbarbati A. In vivo quantitative hydrolipidic map of perirenal adipose tissue by chemical shift imaging at 4.7 Tesla. Int $\mathbf{J}$ Obes Relat Metab Disord 2001;25:457-61.

9. Mosconi E, Fontanella M, Sima DM, Van Huffel S, Fiorini S, Sbarbati A, et al. Investigation of adipose tissues in Zucker rats using in vivo and ex vivo magnetic resonance spectroscopy. J Lipid Res 2011; 52:330-6.

10. Chamosa M. Liposuction of the kneecap area. Plast Reconstr Surg 1997;99:1433-6.

11. Markman B, Barton FE. Anatomy of the 
subcutaneous tissue of the trunk and lower extremity. Plast Reconstr Surg 1987; 80:248-54.

12. Illouz YG. Study of subcutaneous fat. Aesth Plast Surg 1990;14:165-77.

13. Hudson DA, Lambert EV, Bloch CE. Site selection for fat autotransplantation: some observations. Aesth Plast Surg 1990;14: 195-7.

14. Sieg P, Hakim SG, Bierwolf S, Hermes D. Subcutaneous fat layer in different donor regions used for harvesting microvascular soft tissue flaps in slender and adipose patients. Int J Oral Maxillofac Surg 2003; 32:544-7.

15. Sbarbati A, Accorsi D, Benati D, Marchetti L, Orsini G, Rigotti G, et al. Subcutaneous adipose tissue classification. Eur J Histochem 2010;54:e48.

16. Sbarbati A, Galiè M. The niche theory for fat graft survival. In: Fat injection. From filling to regeneration. Coleman SR and Mazzola RF eds. Quality Medical Publishing, Inc., St. Louis, MI, USA, 2009, pp 1530.

17. De Maeseneer M, Gosselin R, De Ridder F,
Shahabpour M, Vanderdood K. MR imaging changes in the trochanteric area of asymptomatic individuals: a potential for misdiagnosis of pain in the trochanteric region. Eur J Radiol 2009;72:480-2.

18. Raskin BI. The art of facial lipoaugmentation. In: Autologous Fat Transfer: Art, Science, and Clinical Practice Shiffman M.A. Ed. 2009. Springer Verlag, Berlin, Germany, pp. 79-86.

19. Galiè M, Konstantinidou G, Peroni D, Scambi I, Marchini C, Lisi V, et al. Mesenchymal stem cells share molecular signature with mesenchymal tumor cells and favor early tumor growth in syngeneic mice. Oncogene 2008;27:2542-51.

20. Peroni D, Scambi I, Pasini A, Lisi V, Bifari F, Krampera M, et al. Stem molecular signature of adipose-derived stromal cells. Exp Cell Res. 2008;314:603-15.

21. Cinti S. The adipose organ. Editrice Kurtis, Milano, Italy, 1999, pp. 1-44.

22. Krampera M, Marconi S, Pasini A, Galiè M, Rigotti G, Mosna F, et al. Induction of neural-like differentiation in human mesenchymal stem cells derived from bone marrow, fat, spleen and thymus. Bone 2007;40:382-90.

23. Anghileri E, Marconi S, Pignatelli A, Cifelli P, Galié M, Sbarbati A, et al. Neuronal differentiation potential of human adiposederived mesenchymal stem cells. Stem Cells Dev 2008;17:909-16.

24. Rigotti G, Marchi A, Stringhini P, Baroni G, Galiè M, Molino AM, et al. Determining the oncological risk of autologous lipoaspirate grafting for post-mastectomy breast reconstruction. Aesthetic Plast Surg 2010;34: 475-80.

25. Galiè M, Pignatti M, Scambi I, Sbarbati A, Rigotti G. Comparison of different centrifugation protocols for the best yield of adipose-derived stromal cells from lipoaspirates. Plast Reconstr Surg 2008; 122: 233e-234e.

26. Rigotti G, Marchi A, Sbarbati A. Adiposederived mesenchymal stem cells: past, present, and future. Aesthetic Plast Surg 2009;33:271-3. 